\title{
Assessment Method for Rolling Bearing Performance Degradation Using TESPAR and GMM
}

\author{
Long Zhang, Wen-yi Huang* , Guo-liang Xiong and Ji-hui Zhou
}

East China Jiao tong University, Nanchang, 330013, China

\begin{abstract}
Rolling bearing performance degradation assessment has been receiving much attention for which itscrucial role to realize CBM(condition-based maintenance). This paper proposed a novel bearing performance degradation method based on TESPAR(Time Encoded Signal Processing and Recognition)and GMM(Gauss Mixture Model). TESPAR is used to extracted features which constitute A-matrix. GMM is utilized to approximate the density distribution of singular values decomposed by A-matrix. TENLLP(Time-Encoded Negative Log Likelihood Probability) serves as a fault severity which can display the similarity of the singular values between normal samples and fault samples as quantificational. Results of its application to bearing fatigue test show that this performance degradation assessment can detect the incipient rolling bearing fault and be sensitive to the change of fault.
\end{abstract}

Keywords: GMM, performance degradation assessment, rolling bearing, TESPAR.

\section{INTRODUCTION}

Bearing failure is one of the foremost causes of breakdowns in rotating machinery and the failure may influence the safety of life and property. Thus the proposed efficient maintenance decision of bearing is a significant issue which cannot be ignored.

CBM (Condition-Based Maintenance) is a crucial maintenance decision being put forward in recent years which is able to avoid the occurrence of insufficient and excessive repairing and rolling bearing performance degradation assessment is the fundamental to realize CBM. To date, the techniques of monitoring the operation of bearing for incipient warning of defects can be classified into three domains: frequency domain analysis, time domain analysis and time-frequency domain analysis. These methods are designed to look for the useful features for diagnosing the failure types or the residual life of the faulted bearing. Fault classification is actually pattern recognition for different bearing faults such as inner fault, out race fault and rolling element fault etc. Various useful methods such as BP neural network, SVM(Support Vector Machine), HMM(Hidden Markov Model) are applied to classify the fault types after extracting features from the signal.

With the increase of the maintenance requirement, performance degradation assessment has drawn more attention than model classification since it is more efficient to realize CBM. Recently, an increasing number of scholars have studied different methods on bearing performance degradation assessment and most of them have made certain achievements. For example, Yan and co-workers [1,2] realize machine performance assessment based on logistic regression. Baydar and Ball [3] analyses the time-frequency diagram of different tooth wear degree under different load based on IPS(Instantaneous Power Spectrum), which found IPS is able to distinguish different fault degree. Huang [4] further predicted the rolling element residual life using a novel method based on back propagation neural network. Stander and Heyns [5] extracted time-frequency features to calculate the Mahalanobis distance between normal samples and fault samples. Wang [6] combines HHT and SOM to realize the bearing performance quantitative classification and assessment. Though these methods get certain achievements, there are still some issues to overcome, such as: the index based on logistic regression is intuitionistic enough, but it needs vibration data of the whole life of the system for knowing their degradation degree. The calculation of Mahalanobis distance is not stable because of the covariance matrix. HHT does not have a specific physical meaning. The result based on Cerebellar Model Articulation Controller Neural Network is seriously influenced by some parameters defined by user, which makes it unpractical.

TESPAR describes signal waveforms in regard to its shapes by coding the signals and putting them in different number to construct $\mathrm{S}$ matrix or A matrix. GMM is an efficient method to fit the signal feature sets into multi-GM smoothly, which is able to model the data with complicated distribution. Thus, GMM is widely used in fields of different precision such as gender recognition, based on speech, and image recognition $[7,8]$. In the field of fault diagnosis, a few of scholars have used GMM in the fault pattern recognition [9] but fewer in performance degradation assessment. This paper proposed a bearing performance degradation assessment method based on TESPAR and GMM. Extracting features from TESPAR and combining them with GMM to describe the distribution, serving the similarity degree of normal bearing feature distribution and fault one's as the fault severity index. To validate the efficiency of this 
approach, bearing fatigue test was applied. Results show that the index based on TESPAR and GMM is able to detect incipient bearing fault and can trace the fault progression well.

\section{TECHNICAL BACKGROUND}

\subsection{TESPAR}

TESPAR is a signal analysis approach derived from the symbolic dynamic theory, chaotic time series analysis and information theory [10]. By dividing the continuous data state space into amounts of discrete cell and gives each cell a number symbol, which transforms the complex data into symbols sequence flow. The signal characteristics of large scale data are able to be captured for reducing the dynamics noise impact in this method $[11,12]$.

\subsubsection{Signal Zero Waveform Parameters}

Digital analysis of TESPAR is to encode the signal cell limited by two adjacent zero. Each cell can be described by two parameters: D (duration-the number of sample signal in cell) and $\mathrm{S}$ (shape-the number of minima sample in positive cell or the number of maxima sample in negative cell). Suppose the signal can be decomposed into $\mathrm{N}$ cells, then it can be composed of $2 \times \mathrm{N}$ parameters. The cell decomposition and cell parameter definition is shown in Fig. (1).

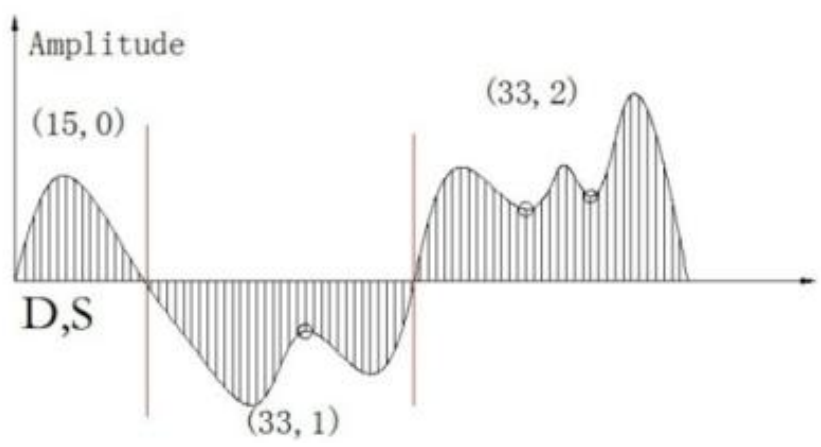

Fig. (1). TESPAR decomposition.

As shown in Fig. (2), the signal can be composed of 3 cells, the [D, S] groups of these cells are: [15,0], [33,1], $[33,2]$, thus the signal can be composed of 6 parameters.

\subsubsection{TESPAR Symbol Table}

In fact, most of the [D, S] groups can be digitally encoded through the corresponding TESPAR symbol table, which makes the original signal transform into a set of digital TESPAR symbol stream. The TESPAR symbol is a kind of experience formula to make the signal within the bandwidth significant, so the TESPAR symbol table can describe any signal cell within the signal bandwidth [13]. TESPAR symbol table is shown in Table $\mathbf{1}$.

\subsubsection{A Matrix}

A matrix is defined as: record the number of each pair of symbols that appears in the TESPAR signal symbol stream. The pair of symbols means the two symbols apart by one symbol. Suppose the TESPAR symbol stream is: $5,8,6,7,5,13,6,2,8,5,7,5,10,6,1$. Looking through the stream which is noted that $(5, \ldots, 6)$ appears 3 times, $(8, \ldots, 7)$ appears
Table 1. TESPAR symbol table.

\begin{tabular}{|c|c|c|c|c|c|c|}
\hline \multirow{2}{*}{ D } & \multicolumn{6}{|c|}{$\mathbf{S}$} \\
\hline & 0 & 1 & 2 & 3 & 4 & 5 \\
\hline 1 & 1 & 1 & 1 & 1 & 1 & 1 \\
\hline 2 & 2 & 2 & 2 & 2 & 2 & 2 \\
\hline 3 & 3 & 3 & 3 & 3 & 3 & 3 \\
\hline 4 & 4 & 4 & 4 & 4 & 4 & 4 \\
\hline 5 & 5 & 5 & 5 & 5 & 5 & 5 \\
\hline 6 & 6 & 6 & 6 & 6 & 6 & 6 \\
\hline 7 & 6 & 6 & 6 & 6 & 6 & 6 \\
\hline 8 & 7 & 8 & 8 & 8 & 8 & 8 \\
\hline 9 & 7 & 8 & 8 & 8 & 8 & 8 \\
\hline 10 & 7 & 8 & 8 & 8 & 8 & 8 \\
\hline 11 & 9 & 10 & 10 & 10 & 10 & 10 \\
\hline 12 & 9 & 10 & 10 & 10 & 10 & 10 \\
\hline 13 & 9 & 10 & 10 & 10 & 10 & 10 \\
\hline 14 & 11 & 12 & 13 & 13 & 13 & 13 \\
\hline 15 & 11 & 12 & 13 & 13 & 13 & 13 \\
\hline 16 & 11 & 12 & 13 & 13 & 13 & 13 \\
\hline 17 & 11 & 12 & 13 & 13 & 13 & 13 \\
\hline 18 & 11 & 12 & 13 & 13 & 13 & 13 \\
\hline 19 & 14 & 15 & 16 & 17 & 17 & 17 \\
\hline 20 & 14 & 15 & 16 & 17 & 17 & 17 \\
\hline 21 & 14 & 15 & 16 & 17 & 17 & 17 \\
\hline 22 & 14 & 15 & 16 & 17 & 17 & 17 \\
\hline 23 & 14 & 15 & 16 & 17 & 17 & 17 \\
\hline 24 & 18 & 19 & 20 & 21 & 22 & 22 \\
\hline 25 & 18 & 19 & 20 & 21 & 22 & 22 \\
\hline 26 & 18 & 19 & 20 & 21 & 22 & 22 \\
\hline 27 & 18 & 19 & 20 & 21 & 22 & 22 \\
\hline 28 & 18 & 19 & 20 & 21 & 22 & 22 \\
\hline 29 & 18 & 19 & 20 & 21 & 22 & 22 \\
\hline 30 & 18 & 19 & 20 & 21 & 22 & 22 \\
\hline 31 & 23 & 24 & 25 & 26 & 27 & 28 \\
\hline 32 & 23 & 24 & 25 & 26 & 27 & 28 \\
\hline 33 & 23 & 24 & 25 & 26 & 27 & 28 \\
\hline 34 & 23 & 24 & 25 & 26 & 27 & 28 \\
\hline 35 & 23 & 24 & 25 & 26 & 27 & 28 \\
\hline 36 & 23 & 24 & 25 & 26 & 27 & 28 \\
\hline 37 & 23 & 24 & 25 & 26 & 27 & 28 \\
\hline
\end{tabular}


twice etc. All other ordinates on the matrix will be zero since there are no more pairings. Here an A matrix has a square base, $13 \times 13$, the height of the ordinate at $(5, \ldots, 6)$ is 3 , at $(8, \ldots, 7)$ is 2 etc. The A matrix bar chart is shown in Fig. (2).

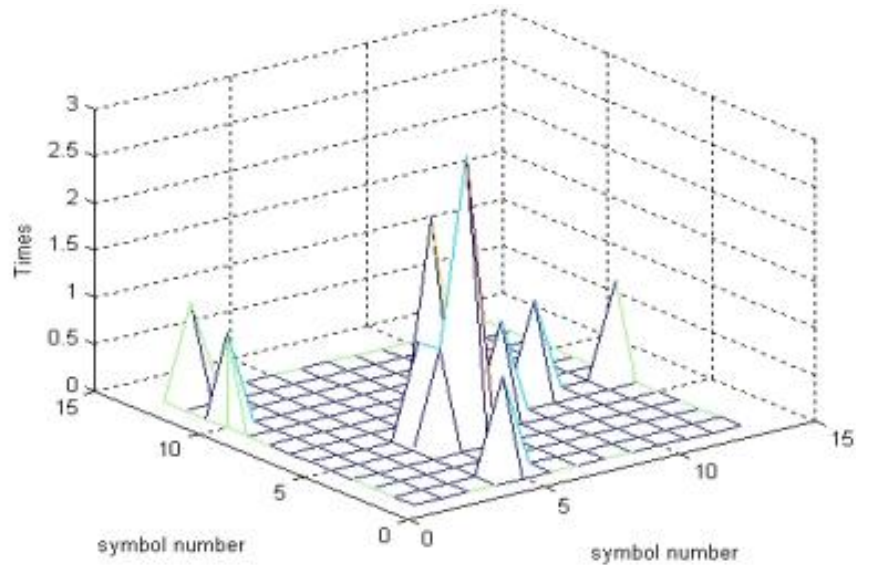

Fig. (2). A matrix bar chart.

\subsection{SVD}

In linear algebra, the singular value decomposition (SVD) is a factorization of a real or complex matrix, with many useful applications in signal processing and statistics.

The singular values arrangement from large to small in the rectangular diagonal matrix $\Sigma$ which is similar to eigenvalue. Generally, the first $10 \%$ or even $1 \%$ singular values occupy the $99 \%$ of the sum. In other words, the first $r$ singular values can be used to describe the $M$ matrix since they contain most information about the structure of the matrix.

\subsection{GMM}

Suppose the data points are approximate ellipsoid distribution in the high dimensional space, then the single Gauss density function can be used to describe the probability density function of these data. But it is not enough to accurately describe the non- elliptical distribution using the single Gauss density function. Gauss Mixture Model (GMM) is an efficient way to solve this issue where it combines a plurality of single Gauss probability density function $\mathrm{P}(x)$ by weighted average to describe the nonelliptical distribution data.

It can be sum up from the GMM formula that the parameters of GMM include $\mathrm{w}_{\mathrm{k}}, \mu_{\mathrm{k}}$ and $\sum_{\mathrm{k}}$, which can be estimated by EM(Expectation Maximum) algorithm [14].

\section{ASSESSMENT METHOD}

In fact, the relative position of input data distribution in Gauss mixture model and the trend of changes in shape can be represented by the mean $\mu_{\mathrm{k}}$ and the covariance matrix $\sum_{\mathrm{k}}$. Thus GMM can approximate any shape density smoothly, which makes the distribution of new data more reasonable and effective than single Gauss model. For the features of vibration signal between normal bearing and fault ones have a certain difference like the A matrix, which is shown in Fig. (3), the GMM is able to describe the feature distribution of normal and fault bearing clearly. This paper proposes the rolling bearing performance degradation assessment model and index by using TESPAR features and GMM.

\subsection{TENLLP}

Construct the GMM as the quantitative baseline for the online monitoring of bearing health status by using the features of normal bearing. Calculate the probability density $\mathrm{p}\left(\mathrm{X}_{\mathrm{i}}\right)$ of the new input data $\mathrm{X}_{\mathrm{i}}$ to represent the probability of input data belonging to the GMM, which consist of healthy signal features. The $\mathrm{P}\left(\mathrm{X}_{\mathrm{j}}\right)$ of the training data $\mathrm{X}_{\mathrm{j}}$ in the input space should be greater than or equal to the threshold and the fault data should be below the threshold. Therefore, the log likelihood probability [15] based on the TESPAR feature (TELLP) is capableof beingused as a bearing performance degradation index. In general, the log likelihood value is less than zero, thus the negative log likelihood probability (TENLLP) is proposed as the quantitative indicator in order to improve its intelligibility:

(a)

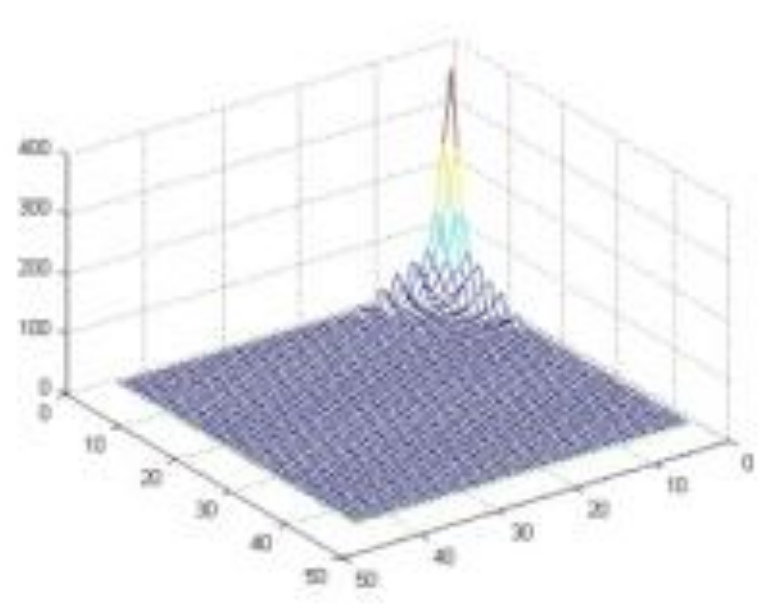

(b)

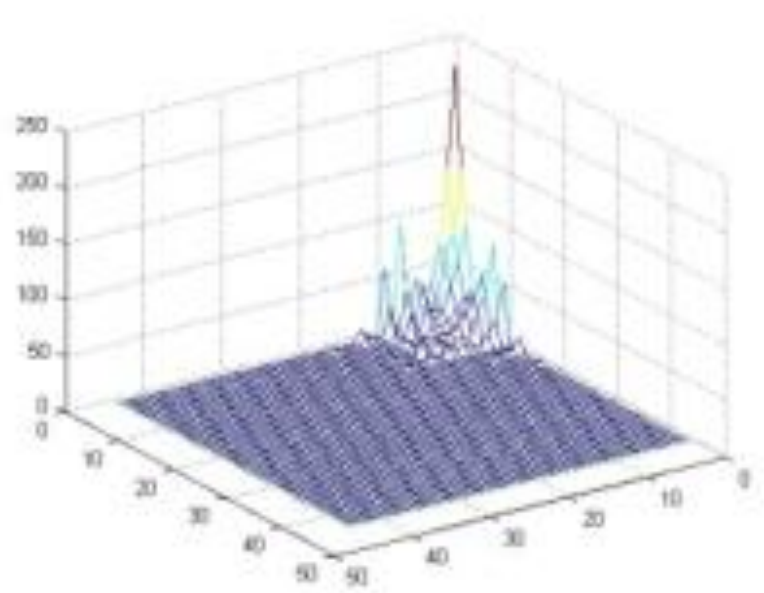

Fig. (3). A matrix of bearing. (a) Normal (b) Fault.

TENLLP $=-\log \left(\mathrm{p}\left(\mathrm{X}_{i}\right)\right)$

\subsection{Model Construction and Index Extraction}

Suppose the early normal bearing data (training data) is $X_{j}(j=1,2,3, \ldots, J)$, the later evaluated data is $X_{i}$, then the 
overall process flow of quantitative performance degradation assessment is shown in Fig. (4).

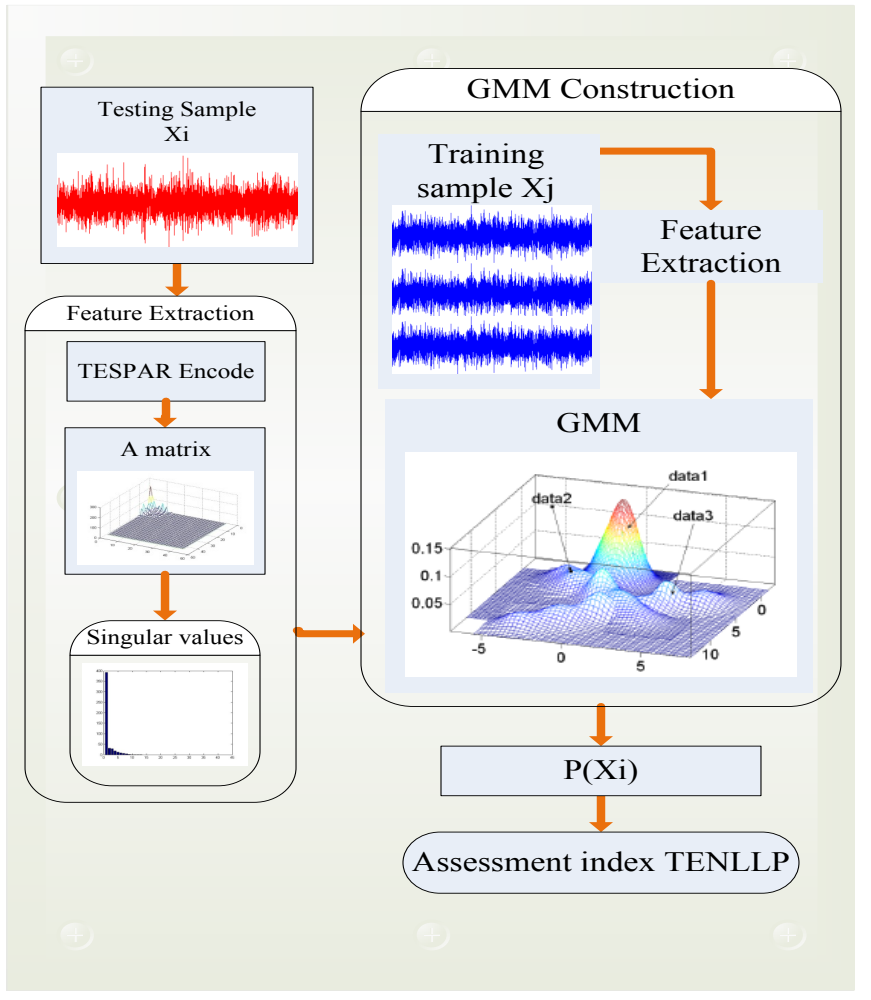

Fig. (4). Assessment process flow.

\section{EXPERIMENT VALIDATION}

To validate the effectiveness of the proposed method, two experiments are applied in this study: bearing fault severity classification experiment and bearing fatigue experiment. The first test is to show the effective classification of TESPAR features for different bearing fault degrees, the other one is to show the ability of proposed index to give the early warning of the bearing defect and the sensibility to the change of degradation in the continuous operation.

\subsection{Fault Severity Classification}

\subsubsection{Experiment Introduction}

This experiment data was downloaded from the Case Western Reserve University bearing data center. As shown in Fig. (5), the experimental device was composed of 2 HP motor (left), a torque sensor, encoder (Center), a dynamometer (right) and the electrical control (not shown). The electric spark technology was adopted to make the defects diameter with dimensions of $0.007 \mathrm{~mm}, 0.014 \mathrm{~mm}$, $0.021 \mathrm{~mm}$ and $0.028 \mathrm{~mm}$ on the SKF bearing on the inner ring, outer ring fault and roller, then acceleration sensor was used to acquire vibration data. This experiment adopted the inner race fault signal and considers that defect of $0.007 \mathrm{~mm}$ to $0.014 \mathrm{~mm}$ belongs to the slight degradation and $0.021 \mathrm{~mm}$ to $0.028 \mathrm{~mm}$ belongs to the severe degradation. The three signals of different fault degree are shown in Fig. (6).

As shown in Fig. (8), the singular values of different degradation degree can be distinguished clearly and with the degradation increase, the first singular value of A matrix increase rapidly.

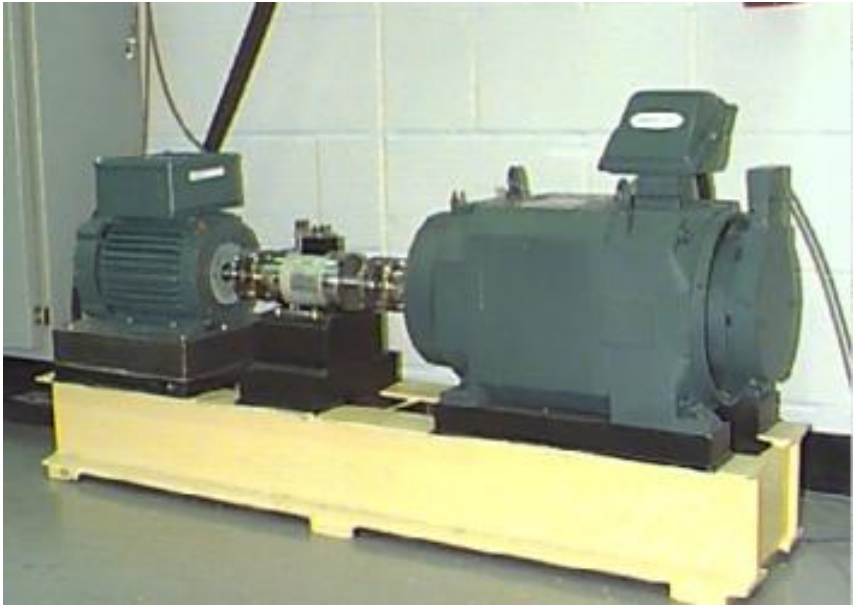

Fig. (5). Bearing fault simulation experiment table.
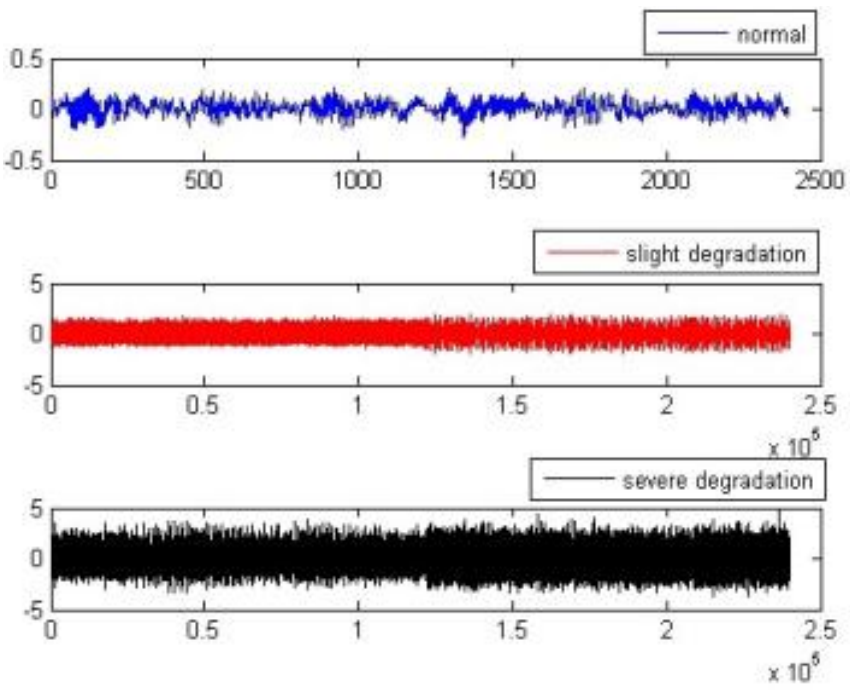

Fig. (6). Three fault degree of inner race.

\subsubsection{Result Analysis}

To authenticate the effective classification of TESPAR singular values, each three groups of experimental signals are divided into 30 columns. Construct the A matrix of each column which is shown in Fig. (7) and calculate the singular values. The first two singular values are shown in Fig. (8).

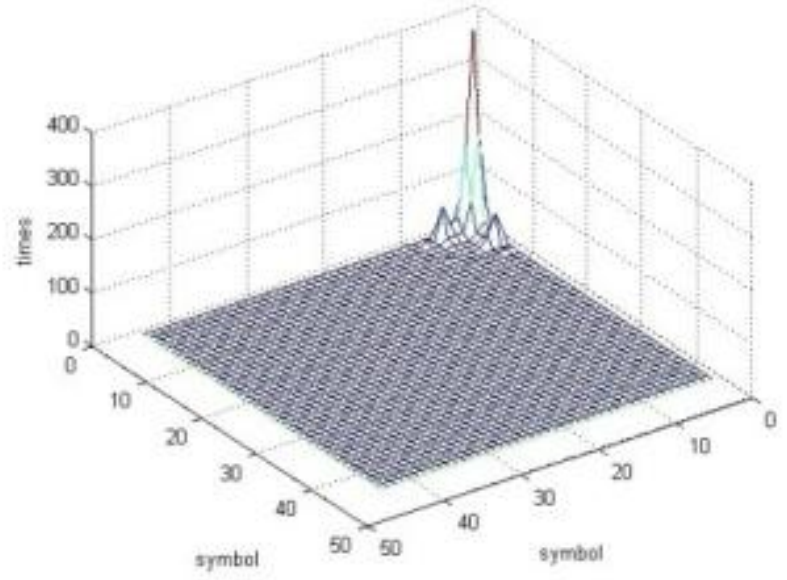

Fig. (7). A matrix of one column. 


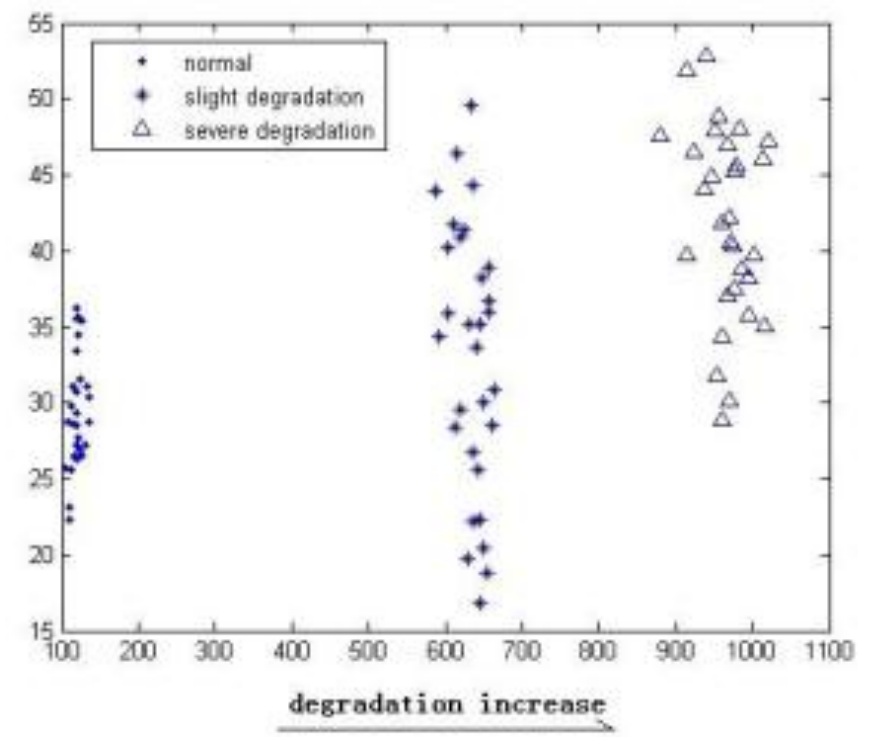

Fig. (8). First two singular values of three different fault degree.

Thus, the singular values of A matrix is served as the extraction feature attributed to its effective classification.

\subsection{Performance Degradation Assessment}

\subsubsection{Experiment Introduction}

This experiment performed bearing run-to failure tests under constant load conditions on a specially designed test rig as shown in Fig. (9) [16]. The bearing test rig host's four test Rexnord ZA-2115 double row bearing on one shaft. The shaft was driven by an AC motor and coupled by rub belts. The rotation speed was kept constant at $2000 \mathrm{rpm}$. A radial load of $6000 \mathrm{lbs}$ was added to the shaft and bearing by a spring mechanism (Fig. 10).

Data collection started form the 2013.2.12 10:32:39 to 2013.2.19 06:22:39, collect the vibration signals every 10 minutes during acquisition time, 984 data files were altogether collected during the experimental process. The sampling frequency is $2000 \mathrm{~Hz}$, each sensor collect 20480 data each time, this paper analysis 8192 data of the second file (Bearing 2).

\subsubsection{Model Construction and Result Analysis}

Pick the first 6 A matrix singular values of all 984 files to construct a feature matrix of $6 \times 984$ which is shown in Fig. (11) and select the features of first 100 data file as the training sample to construct the normal Gauss mixture model. Then regard all the features as the test sample which are substituted in the GMM and obtain the TENLLP of each file and are plotted in Fig. (11).

From the Fig. (11), it is not easy to observe the process of degradation by naked eye and that makes GMM outstanding in distinguishing the change of fault degree.

Also it can be concluded from the Fig. (11) that: (1) the process of bearing performance degradation ranges from normal, slight degradation, severe degradation and failure; (2) the duration of slight degradation is longer than the severe, so it is reasonable to take steps of maintenance before (a)

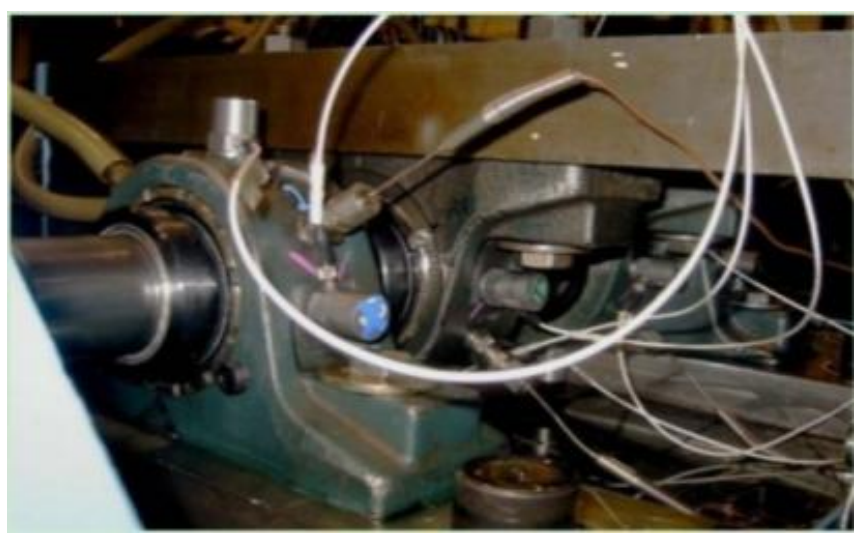

(b)

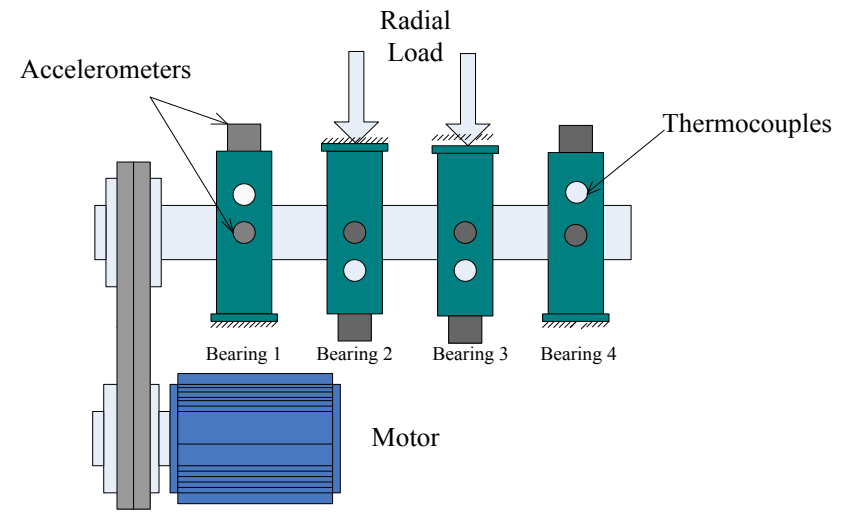

Fig. (9). Bearing fatigue test. (a) Test bench (b) Sketch map of bench.

the bearing runs into the severe degradation; (3) TENLLP is an effective index to give alarm in early appearance of fault and it has the advantages of sensitivity and accuracy for bearing performance degradation degree.

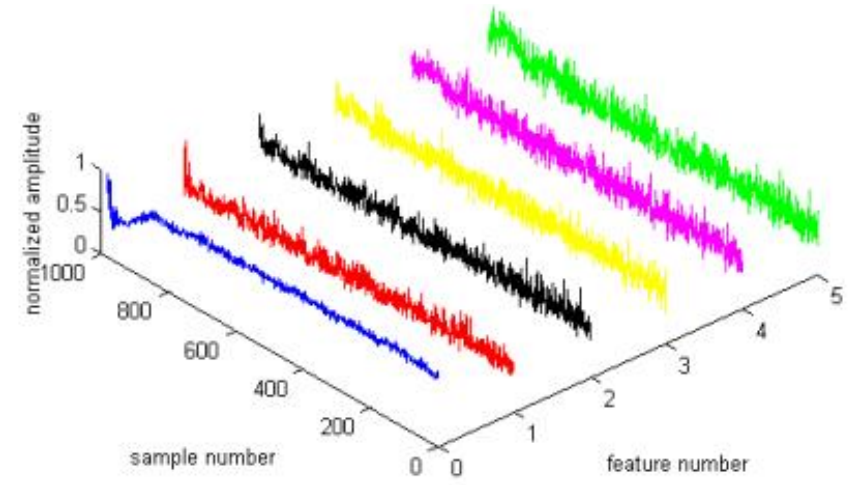

Fig. (10). Singular values of all samples.

\subsubsection{Compared with the Previous Index}

Kurtosis has been an important indicator in the detection of bearing fault degree diagnosis, the kurtosis result of the experimental data is shown in Fig. (12).

Obviously, Compared with this method, TENLLP can clearly present the degradation of bearing failure, and can give warning in early appearance of fault. 


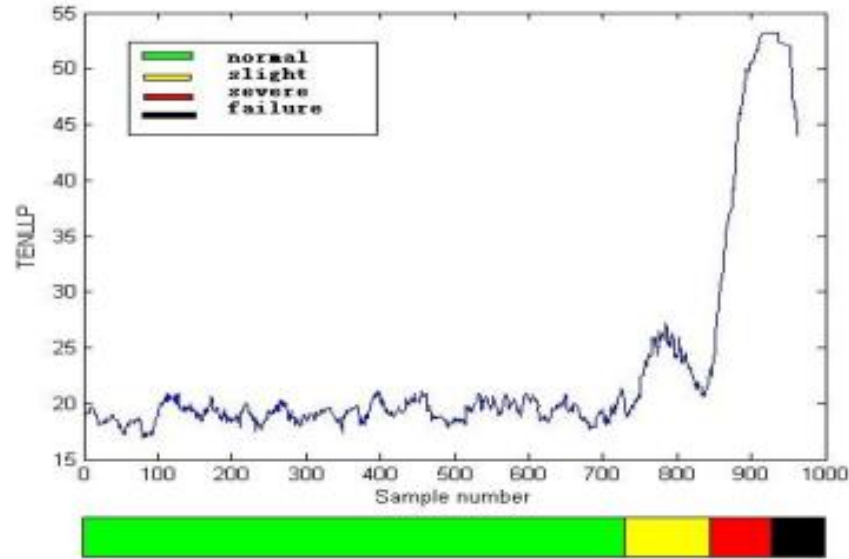

Fig. (11). TENLLP of all samples.

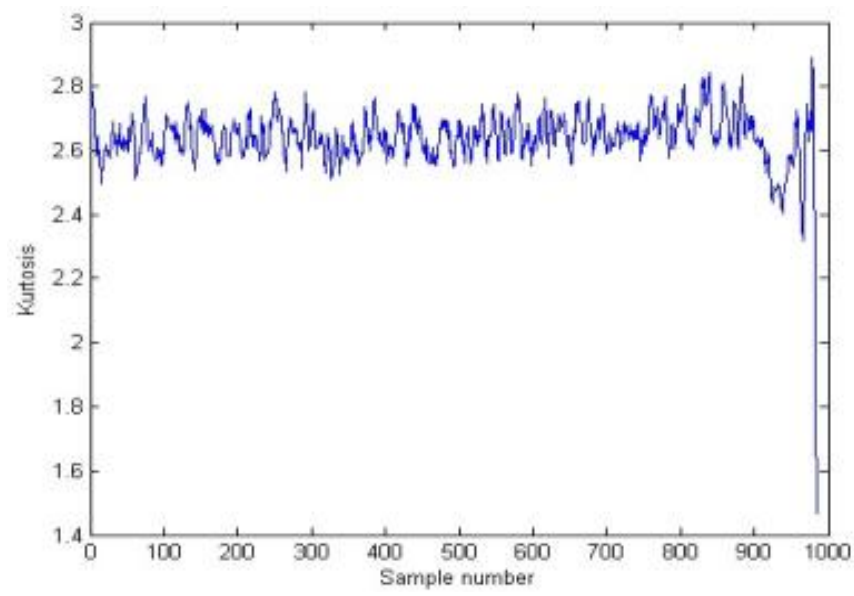

Fig. (12). Kurtosis of all samples.

\section{CONCLUSION}

In this paper, the quantitative assessment of rolling bearing performance degradation based on the TESPAR and GMM is proposed, which serves the TENLLP as the fault severity index. A novel FE approach, singular values of A matrix based on TESPAR is developed and the fault severity classification experiment validates the effective classification offault degree of this feature. The bearing fatigue test experiment based on this new feature and GMM shows that the combinative index TENLLP is able to recognize the slight degradation of bearing in an early stage and is highly sensitive to the change of degradation level on its whole life. From the above, this new approach of degradation assessment has a strong practical applicationfor rolling bearing fault degree monitoring.

\section{CONFLICT OF INTEREST}

The authors confirm that this article content has no conflict of interest.

\section{ACKNOWLEDGEMENTS}

This work was financially supported by the Natural Science Foundation of China $(51205130,51265010) \&$ the Foundation of Jiangxi Educational Committee (GJJ12318) as well as the Natural Science Foundation of Jiangxi Province (20132BAB216029).

\section{REFERENCES}

[1] J.H. Yan, and J. Lee, "Degradation assessment and fault modes classification using logistic regression", Journal of Manufacturing Science and Engineering-Transactions of the ASME, vol. 12, pp. 912-4, 2005.

[2] J. Yan, M. Koc, and J. Lee, "A prognostic algorithm for machine performance assessment and its application", Production Planning and control vol. 15, pp. 796-801, 2004

[3] N. Baydar, and A. Ball, "Detection of gear failures via vibration and acoustic signals using wavelet transform", Mechanical Systems and Signal Processing, vol.17, pp. 787-804, 2003.

[4] R.Q. Huang, L.F. Xi, X.L. Li, C.R. Liu, H. Qiu, and J. Lee, "Residual life predictions for ball bearing based on self-organizing map and back propagation neural network methods", Mechanical Systems and Signal Processing, vol. 21, pp. 193-207, 2007.

[5] C. J. Stander, P. S. Heyns, and W. Schoombie, " Using vibration monitoring for local fault detection on gears operating under fluctuating load conditions", Mechanical Systems and Signal Processing, vol.16, pp. 1005-24, 2002.

[6] W. Jianli, "Research on performance degradation assessment and trend prediction of rolling bearing",Dalian: Dalian University of Technology, 2013.

[7] Z. Chaoqiong, and M. D. Yue Xiaodong, "Speech gender recognition based on Gauss mixture model",Computer Applications, vol. 28, pp. 360-5, 2008.

[8] C. Xuefeng, and J. Yunde, "Discriminative learning approach for Gaussian Mixture modeling for images", Beijing Institute of Technology, 2009.

[9] X. Han, L. Yourong, and L. Yong, "Gear fault recognition based on recurrence quantification analysis and gauss mixture model", Journal of Vibration Engineering, vol. 1, pp. 84-8, 2011.

[10] Q. Guojun, M. Yuanyuan, and H. Niaoqing, "A research on time encoded signal processing for real-time fault detection of turbo pump", Journal of National University of Defense Technology, vol. 30, pp. 104-6, 2008.

[11] S. Abdusslam, P. Raharjo, F. Gu, and A. Ball, "Bearing defect detection and diagnosis using a time encoded signal processing and pattern recognition method", Journal of Physics, vol. 364,pp. 012036, 2012.

[12] S. Abdusslam, M. Ahmed, P Raharjo, F. Gu, and A. D. Ball. Time encoded signal processing and recognition of incipient bearing faults", Proceedings of the 17th International Conference on Automation \& Computing, University of Huddersfield, Huddersfield, UK, 10 September 2011

[13] S. A. Abdusslam, "Detection and diagnosis of rolling element bearing faults using time encoded signal processing and recognition”, University of Huddersfield, 2012.

[14] A. C. Lindgren, M. T Johnson, and R.J. Povinelli, Speech space features. Acoustics, Speech, and Signal Processing, 2003 Proceedings (ICASSP 03), IEEE International Conference, Hong Kong, China, 2003: I-60-3.

[15] J. Yu, "Bearing performance degradation assessment using locality preserving projections and Gaussian mixture models", Mechanical Systems and Signal Processing, vol. 25, pp. 2573-88, 2011.

[16] J. Lee , H. Qiu , G. Yu and J. Lin Rexnord Technical Services: Bearing Data Set, 2007 :IMS, Univ. Cincinnati. NASA Ames Prognostics Data Repository, NASA Ames [online] Available. 